\title{
Bioanalysis
}

\section{Microscale bioanalysis}

\section{"Over the last couple of years, tremendous technology development has opened an array of new opportunities for several categories of microscale bioanalytical tools in drug development."}

\section{First draft submitted: 9 March 2016; Accepted for publication: 31 March 2016; Published online: 25 April 2016}

\section{Keywords: bioanalysis $\bullet$ drug development $\bullet$ imaging $\bullet$ microfluidics $\bullet$ microsampling} - microseparations • organs-on-a-chip

While various microscale bioanalytical techniques have been used for a long time in the academic/research arena, it has always taken some time for these innovations to reach the drug development sphere of bioanalysis. There are multiple reasons for this slow adaptation of new techniques in drug development, including the need for sufficiently robust and easy-to-use tools in a routine bioanalytical environment, and an inborn desire of the bioanalytical scientist to ensure compliance of the analysis with regulatory standards.

Over the last couple of years, tremendous technology development has opened an array of new opportunities for several categories of microscale bioanalytical tools in drug development. In this article, we want to make the distinction between the following three main categories: microsampling; microseparations; and, less obvious, imaging.

Micro total analytical systems ( $\mu$ TAS) or lab-on-a-chip (LOC) microfluidic technologies are well-suited for drug discovery and development due to the many advantages of the technology [1]. For example, microfluidics requires small amounts of reagent and sample volumes, thereby, resulting in a reduction in costs, parallel processing of samples in less time, and improvement in data quality and performance. These advantages allow for unique capabilities in sample preparation and separation, combinatorial synthesis, and preclinical testing of drugs in living cells required in drug development. Microfluidic technologies are powerful tools for drug discovery and development processes and has great potential in a number of areas. In the pharmaceutical industry, applications can be founds in areas of diagnostics and drug research. These applications include target selection, lead identification, optimization and preclinical tests, chemical synthesis, clinical trials, formulation studies, and product management processes $[2,3]$.

\section{Microsampling}

Since the introduction of DBS as a blood collection tool for TK/PK some years ago [4] there have been numerous articles $[5,6]$ including a special edition of Bioanalysis [7] of applications of this technique in the regulated bioanalysis environment. In parallel with researchers trying to overcome some of the shortcomings of DBS, including the hematocrit effect or homogeneity, much focus today is also towards wet microsampling. While the 3R's have been the main driver for these microsampling techniques, the development of latest generation triple quadrupole mass spectrometers has been essential in order to implement these techniques while maintaining overall sensitivity of the assay.

Although less pronounced in the scientific literature and at bioanalytical conferences, the combination of microsampling and ligand binding assay offers great opportunities, just as promising than micro-

\author{
Magnus Knutsson \\ Ferring Pharmaceticals A/S, Bioanalysis \\ Department, Copenhagen, Denmark, \\ Kay Fiskers Plads 11, DK-2300 \\ Copenhagen S, Denmark
}

Philip Timmerman Janssen R\&D, Bioanalysis Department, Turnhoutseweg 30, B-2340 Beerse, Belgium

\section{Frank A Gomez}

Author for correspondence: Department of Chemistry and Biochemistry, California State University, Los Angeles, 5151 State University Drive, Los Angeles, California 90032-8202, USA

Tel.: +1 3233432368

Fax: +1323 3436490

fgomez2@calstatela.edu 
sampling and conventional size LC-MS, i.e., 2.1-mm inner diameter. The smaller volumes in microsampling naturally fits the volumes normally used in various ligand binding assay formats, and this may be the explanation of the lesser focus in peer-reviewed literature.

\section{Microseparations}

It is important to distinguish between 'microsampling' and 'micro- (or nano-) separations'. Whereas the former focuses on the ethical advantages of needing smaller sample volumes (3Rs, neonates or scarce matrices) or enabling sampling for those situations where no sample would have been possible using standard sampling, the latter enables by the developments in separation technology, both front end (e.g., chromatography) and detector (e.g., hyphenation, MS, TOF or imaging). Many tools have been used for numerous years within the field of proteomics research $[8,9]$. Its introduction to the drug development bioanalytical field has taken some time and as only recently has more robust and easy-to-use microchromatographic tools and micro-MS systems allowed for more widespread use of this technique [10-12].

We would like to focus on two main interesting application tools of microscale separations in drug development bioanalysis;

1. Microscale separations in combination with various microsampling: for example, dry or wet microsampling will benefit from the potential of (increased) sensitivity which is often the result of assays involving microseparations;

2. Microscale separations in support of peptide and protein quantification: the increased ionization efficiency which the low flows facilitate gives an even more pronounced MS sensitivity for peptides and proteins and thus this type of applications gains more and more interest, which is also seen in some of the papers in this special edition $[13,14]$.

Typically, for these two categories, column dimensions used for microseparations in drug development bioanalysis are around $150 \mu \mathrm{m}$, seeking a compromise between sensitivity, retention times and assay robustness. The resulting assays are becoming more and more part of the standard toolbox of the bioanalytical scientist. Smaller column diameters (e.g., $75 \mu \mathrm{m}$ ) are subject of intense testing by some expert groups. Smaller column diameters mean smaller injection volumes to fully benefit from the better ionization and increased sensitivity.

In order to maintain the overall assay sensitivity in either micro- or nano-scale separations, some research groups combine the injection larger volumes, either on trap-elute systems $[15,16]$ or multidimensional chromatographic system with columns with varying inner diameter, for example, injecting $50 \mu \mathrm{l}$ from $2.1 \mathrm{~mm}$ i.d. and in series downscaling the dimension ending up with a $150 \mu \mathrm{m}$ i.d. [17]. The results in this area of micro-/nano-scale separations are promising, but further research and development will be required to increase the robustness of this technology in future years.

\section{Imaging}

A third category we want to focus on very shortly in this paper are microscale separations in support of quantitative $2 \mathrm{D} / 3 \mathrm{D}$ (tissue) imaging. Although less obvious than the previous two categories, increased resolution and better handling of $2 \mathrm{D} / 3 \mathrm{D}$ separations is a new potential area of focus for the enhanced use of microscale technologies [18]. We anticipate an increasing number of applications in quantitative $2 \mathrm{D} / 3 \mathrm{D}$ imaging in early development, providing additional tools to document ADME properties or drugs, metabolites, and biomarkers in the next decade.

\section{Future perspectives on microscale bioanalysis in drug development}

In spite of the relatively slow implementation of microscale bioanalytical tools into drug development, it is foreseen that the technology will gain more and more interest in the years to come in the three above mentioned categories. Not only because it is fun in the lab to play with new tools, but more so because of increased emphasis in drug development on novel therapies, personal medicines, and/or bedside monitoring. Hence, there will be a growing interest to implement novel microtechniques to easily and rapidly monitor, for instance, biomarkers or patient compliance. In order to make this happen, it is important that we approach the challenge with an open and inviting mind. Getting inspired by other scientific communities other than our own small bioanalytical family is important. Outpatient diagnostic tools (e.g., blood sugar monitoring devices in diabetes management) can help bioanalytical scientists 'locked away in their LC-MS lab' to inspire and get inspired by other analytical communities. And, as highlighted in the introduction, no new technology from cradle to production ever resulted without intensive trial and error. In this context, it is important to allow new technologies to improve their performance or to understand the pitfalls and benefits in order not to abandon the tool if it doesn't immediately meet (regulatory inspired) acceptance criteria for a tool or in the development phase [19]. 
Long-term, the drug industry can benefit from utilizing microfluidic technologies in a number of drug development stages including target screening, lead optimization, and toxicity studies in preclinical studies. Furthermore, microfluidics should be a better technology in the production of new chemical products and for their screening against biological targets than current methods. Microfluidics also provides inventive technological opportunities for obtaining new information about biological systems.

\section{Future of microfluidics in drug development: organs-on-a-chip}

Research and development costs in the pharmaceutical industry to develop new drugs is unsustainable. The exclusivity of blockbluster drugs expiring coupled to fewer new drugs being approved has necessitated industry adopt new strategies for drug development to continue to bring new drugs to market. The majority of applied tests for drug discovery are based on a $2 \mathrm{D}$ cell culture model which is easy to handle and automate and can be used for high throughput screening. Such tests do not allow for the simulation of interactions present in vivo thereby limiting their ability to predict the effect of the drug on humans [20]. Hence, increasing attention will be paid to the development of $3 \mathrm{D}$ cellular models in microfluidic platforms that will mimic cell-to-cell interactions that occur in vivo thereby allowing for more facile prediction of the results of drug interactions. Organs-on-a-chip allow for high-resolution, real-time imaging and in vitro analysis of biochemical, genetic and metabolic activities of living cells, in a context of functional tissues and organs, all important aspects of drug development.

Recently, novel microfluidic devices (MDs) have been developed to reproduce in vivo organ function but on a microchip platform. These 'organ-on-a-chip' technologies are based on foundational microfluidic technologies and integrate components into an in vitro device thereby allowing researchers to replicate in vivo function [21]. Organs-on-chip are small MDs with channels that are lined by living human cells. They have properties that make them more realistic models of human organs than typically lab-grown cells. In the MDs, the cells are arranged to mimic structural features of the architecture of the organ. The properties of organs-on-a chip allow scientists to analyze interactions between drugs and cells that are usually only measured in living animals. While still in its infancy, examples of promising studies include kidney-on-a-chip, cancer-on-a-chip, and gut-on-achip. An ultimate goal would be linking these devices (modules) into a human-on-a-chip system. Here, a number of drugs could be tested in a series of in vitro assays that would allow for the prediction of a new drug (hit rate) even before animal testing and human clinical trials are conducted.

For example, recently a MD lined by living human kidney epithelial cells exposed to fluidic flow that mimics key functions of the human kidney proximal tubule was described [22]. Kidney toxicity is known to be one of the most adverse results during drug development. The epithelial monolayer was exposed to a shear stress similar to that found in living kidney tubules and compared with Transwell culture systems. The kidney-on-a-chip was assessed for drug transport and nephrotoxicity and was found to have greater albumin transport, glucose reabsorption. In another study, a MD fabricated from poly(dimethylsiloxane) (PDMS) was developed to mimic the alveolar-capillary interface of a human lung by integration of a mechanical cell actuator into a co-culture model. A two-layer structure was used to co-culture in a central channel while applying vacuum channels on either side thereby generating mechanical actuation [23]. A device such as this delivers results of drug efficacy and toxicity on an organ level and can provide valuable information in future drug development.

A great deal more work is still needed before organon-a-chip techniques can be accepted in drug development but recent advancements in this area are promising. Case in point, AstraZeneca has formed a collaboration with Harvard's Wyss Institute to leverage the institute's technologies to better predict safety of drugs in humans.

This special issue on microscale bioanalysis in drug development brings together the work of scientists and researchers from across multiple disciplines to demonstrate the strong overlap between their efforts and how their work exemplifies the exciting potential of bioanalysis in drug development. As demonstrated, bioanalysis is interdisciplinary encompassing a wide array of analytical techniques and methodologies. It is our goal that this special issue will inspire future scientists to utilize the newest bioanalytical techniques when exploring the frontiers of drug development. Only by bringing together people of varied backgrounds can the field reach its full potential.

\section{Financial \& competing interests disclosure}

The authors have no relevant affiliations or financial involvement with an organization or entity with a financial interest in or financial conflict with the subject matter or materials discussed in the manuscript. This includes employment, consultancies, honoraria, stock ownership or options, expert testimony, grants or patents received or pending, or royalties.

No writing assistance was utilized in the production of this manuscript. 


\section{References}

1 Jalali-Heravi M, Arrastia M, Gomez FA. How chemometrics can improve microfluidic research? Anal. Chem. 87, 3544-3555 (2015).

2 Neuzil P, Giselbrecht S, Lange K, Huang TJ, Manz A. Revisiting lab-on-a-chip technology for drug discovery. Nat. Rev. Drug Discov. 11, 620-632 (2012).

3 Wu M-H, Huang S-B, Lee G-B. Microfluidic cell culture systems for drug research. Lab Chip 10, 939-956 (2010).

4 Beaudette P, Bateman KP. Discovery stage pharmacokinetics using dried blood spots. J. Chromatog. B 809, 153-158 (2004).

5 Timmerman P, White S, Cobb Z, de Vries R, Thomas E, van Baar B. Update of the EBF Recommendation for the use of dried blood spots in regulated bioanalysis towards the conclusions from the EBF DBS micro sampling consortium. Bioanalysis 5(17), 2129-2136 (2013).

6 Spooner N, Lad R, Barfield M. Dried blood spots as a sample collections technique for the determination of pharmacokinetics in clinical studies: considerations for the validation of a quantitative bioanlaytical method. Anal. Chem. 81(4) 1557-1563 (2009).

7 Mini Focus Issue: Dried blood spots. Bioanalysis 5(17) (2013).

8 Ishihama Y. Proteomic LC-MS system using nanoscale liquid chromatography with tandem mass spectrometry. J. Chromatogr. A, 1067(1), 73-83 (2005).

9 Köhler T, Pichler P, De Pra M, Rieux L, Swart R, Mechtler K. Development and performance evaluation of an ultralow flow nanoliquid chromatographic-tandem mass spectrometry set-up. Proteomics 14 (17-18), 1999-2007 (2014).

10 Agilent Technologies, HPLC-Chip Cube MS Interface. www.chem.agilent.com

11 Eksigent cHiPLC systems. www.eksigent.com

12 Waters ionKey/MS system. www.waters.com
13 Wilffert D, Asselman A, Donzelli R et al. Highly sensitive antibody-free $\mu \mathrm{LC}-\mathrm{MS} / \mathrm{MS}$ quantification of rhTRAIL in serum. Bioanalysis 8(9), 881-890 (2016).

14 Kleinnijenhuis A. Quantitative analysis of infliximab in serum using $\mu \mathrm{LC}-\mathrm{MS} / \mathrm{MS}$. Bioanalysis 8(9), 891-904 (2016).

15 Schultz GA, McCardle K, Neubert H. Large-scale implementation of sequential protein and peptide immunoaffinity enrichment LC/nanoLC-MS/MS for human $\beta$-nerve growth factor. Bioanalysis 8(8), 753-764 (2016).

16 Chambers EE, Lame ME, Rainville PD, Murphy J, Johnson J, Fountain KJ, Plumb RS, Claise P, Smith NW. Practical applications of integrated microfluidics for peptide quantification. Bioanalysis 7(7) 857-867 (2015).

17 Lubin A, Vereyken L, de Vries R et al. Ultra high sensitivity bioanalysis by improved ionization and 2D-microUHPLC applying chip technology. Presented at: The 63rd ASMS Conference on Mass Spectrometry. MO, USA, 31 May-4 June 2015.

18 Stauber J. Quantitation by MS imaging: needs and challenges in pharmacueticals. Bioanalysis 4(17), 2095-2098 (2012)

19 Timmerman P, Henderson N, Smeraglia J et al. Managing scientific, technical and regulatory innovation in regulated bioanalysis: a discussion paper from the European Bioanalysis Forum. Bioanalysis 5(2), 139-145 (2013).

20 Kwapiszewska K, Michalczuk A, Rybka M, Kwapiszewski R, Brzózka Z. A microfluidic-based platform for tumour spheroid culture, monitoring and drug screening. Lab Chip 14, 2096-2104 (2014)

21 Bhatia SN, Ingber DE. Microfluidic organs on chips. Nat. Biotechnol. 8, 760-772 (2014).

22 Jang KJ, Mehr AP, Hamilton GA et al. Human kidney proximal tubule-on-a-chip for drug transport and nephrotoxicity assessment. Integr. Biol. 9, 1119-1129 (2013).

23 Huh D, Matthews BD, Mammoto A, Montoya-Zavala M, Hong YH, Ingber DE. Reconstituting organ-level lung functions on a chip. Science 328, 1662-1668 (2010). 